\title{
Anodic Electrochemiluminescence of CdTe Quantum Dots Using Tripropylamine as Coreactant: Size-dependent Effect
}

\author{
Masayuki NAKAYAMA, * Takuma KITANo, * Jianshan Ye,** and Jiye Jin*† \\ *Department of Chemistry, Faculty of Science, Shinshu University, 3-1-1 Asahi, Matsumoto, Nagano 390-8621, \\ Japan \\ **College of Chemistry and Chemical Engineering, Key Laboratory of Fuel Cell Technology of Guangdong \\ Province, South China University of Technology, Guangzhou 510641, P. R. China
}

\begin{abstract}
The quantum size effect of CdTe quantum dots (QDs) has been one of the targets of extensive research concerning the optical spectroscopy of semiconductors, but little is known about their effects on electrochemiluminescence (ECL) behavior, especially in the anodic potential range. In this present study, water-soluble CdTe QDs with different sizes were synthesized with a microwave-assisted hydrothermal method. Upon electrochemical oxidation of the CdTe QD in the presence of tri- $n$-propylamine (TPrA) as a coreactant, two ECL signals, called $\mathrm{ECL}_{1}$ and $\mathrm{ECL}_{2}$, were observed at potentials corresponding to the oxidation of TPrA (at $+0.8 \mathrm{~V}$ ) and CdTe QDs (at $+1.2 \mathrm{~V}$ ), respectively. The relative intensity of ECL $\mathrm{EL}_{1}$ significantly increased with increasing the particle size of CdTe QDs, and disappeared when the particle size was less than $2.4 \mathrm{~nm}$. Upon an anodic potential of $+0.8 \mathrm{~V}$, TPrA is oxidized at the electrode surface, where the intermediate radical species like $\operatorname{TPrA}^{*+}$ radical cation and CdTe QDs.- radical anion are supposed to be formed to give the excited chemical species of CdTe QDs*. Possible ECL mechanisms are proposed from a view point of thermodynamics.
\end{abstract}

Keywords CdTe quantum dots, electrochemiluminescence, tripropylamine, size-dependent, Gibbs free energy, band gap energy

(Received December 26, 2019; Accepted January 24, 2020; Advance Publication Released Online by J-STAGE January 31, 2020)

\section{Introduction}

Semiconductor nanocrystal quantum dots (QDs) have been attracting extensive attention in analytical science because QDs offer a number of advantages for their use in fluorescence probes, such as highly quantum yields and good photo stability as well as narrow-emission spectra that are continuous and tunable due to quantum size effects. ${ }^{1-4}$ In the past two decades, significant advances have been made towards the synthesis of colloidal semiconductor QDs, particularly II-VI compounds, such as cadmium selenide (CdSe), cadmium sulfide (CdS) and cadmium telluride (CdTe). ${ }^{1,2}$ These highly luminescent nanomaterials possess suitable characteristics for various applications in optoelectronics and biological labeling. ${ }^{3-6}$

Water-soluble thiol-capped CdTe QDs have been demonstrated to be one of the most robust and highly fluorescent nanoparticle materials that could be directly synthesized in an aqueous medium. ${ }^{7,8}$ Since they also show electrochemical activity, the electrochemiluminescence (ECL) phenomena of QDs and their practical applications in aqueous media have been extensively studied in recent years. By using coreactants, such as hydrogen peroxide and peroxydisulfate, ${ }^{9-11}$ ECL signals have been observed in the cathodic potential range at a carbon nanotubes modified glassy carbon (GC) electrode and a CdSe QDs immobilized graphite electrode, respectively. In view of the importance of anodic ECL of QDs in bioanalysis, great efforts

† To whom correspondence should be addressed.

E-mail: jin@shinshu-u.ac.jp have focused on reducing the potential and studying the reaction scheme of ECL in an anodic potential range. Liu and co-workers reported an anodic ECL of CdTe QDs in aqueous systems at an ITO electrode with a stable and intensive anodic ECL emission of around $+1.17 \mathrm{~V}$ vs. $\mathrm{Ag} / \mathrm{AgCl} .{ }^{12}$ The ECL emission involved collisions between the superoxide ion produced at the ITO surface and the oxidation products of QDs. Dong and co-workers demonstrated the ECL of CdTe QDs at the anodic potential range using 2-(dibutylamino) ethanol as a coreactant. ${ }^{13}$ It is known that the optical and electrochemical properties exhibit characteristics of size dependence due to their variety of band gap energy $\left(E_{\mathrm{g}}\right)$, but little is known about how QDs particle size affects the response of ECL. There are still many unclear issues concerning the reaction mechanisms in the anodic potential range. In this study, we synthesized CdTe QDs with different particle sizes by a microwave-assisted hydrothermal method. This is the first time to report the size effect of CdTe QDs on anodic ECL in the presence of tripropylamine (TPrA), a well-known coreactant in the $\mathrm{Ru}(\mathrm{bpy})_{3}{ }^{2+}$ ECL system. ${ }^{14-16}$

\section{Materials and Methods}

\section{Chemicals}

Cadmium chloride $\left(\mathrm{CdCl}_{2}\right)$, 3-mercaptopropionic acid (MPA), and tris(hydroxymethyl)aminomethane (Tris) were purchased from Nacalai Tesque (Kyoto, Japan). Sodium tellurite pentahydrate $\left(\mathrm{Na}_{2} \mathrm{TeO}_{3} \cdot 5 \mathrm{H}_{2} \mathrm{O}\right)$ and sodium hydroxide $(\mathrm{NaOH})$ were purchased from Kanto Chemical Co., Inc. (Tokyo, Japan). 
Tri- $n$-propylamine (TPrA), 2-n-propanol, and hydrochloric acid $(\mathrm{HCl})$ were purchased from Wako Pure Chemical Industries, Ltd. (Osaka, Japan); 0.1 M Tris-HCl buffer solution (pH 9) was prepared from a tris(hydroxymethyl)aminomethane solution. The $\mathrm{pH}$ was adjusted by the addition of $0.1 \mathrm{M} \mathrm{HCl}$. Pure water used in all of the experiments was produced by a WS-200 Pure water production system auto still (Yamato Scientific Co., Ltd.).

\section{Apparatus}

Electrochemical and ECL measurements were carried out using a CV-27 potentiostat/galvanostat (BAS Japan) at room temperature, with a configuration consisting of a working electrode, a platinum counter electrode, and an $\mathrm{Ag} / \mathrm{AgCl}$ reference electrode (RE-1, BAS Japan). A glassy carbon disk (Tokai Carbon Co., Tokyo, Japan) with diameter of $3 \mathrm{~mm}$ was used as a working electrode. Before the measurement, the working electrode was polished with $0.3 \mu \mathrm{m}$ of the alumina slurry, sonicated in an ultrasound bath, and finally rinsed with water. The light emission from the electrode surface was measured by a H7732-10 PMT photosensor module equipped with a C7319 signal preamplifier unit (Hamamatsu Photonics, Japan). A C7169 power supply unit (Hamamatsu Photonics, Japan) was used for driving the photosensor module. The electrode surface of the working electrode was at $0.5 \mathrm{~mm}$ distance from the optical window.

\section{Synthesis of colloidal CdTe QDs}

A facile one-pot hydrothermal method was employed for the synthesis of highly luminescent CdTe QDs in an aqueous environment by microwave irradiation, using $\mathrm{Na}_{2} \mathrm{TeO}_{3}$ as the $\mathrm{Te}$ source. This eliminated the need for using highly toxic $\mathrm{H}_{2} \mathrm{Te}$ or highly unstable NaHTe as a precursor for the aqueous synthesis of CdTe QDs under the inert atmospheric conditions. ${ }^{17}$ A fifty milliliter portion of the reaction solution containing $1 \mathrm{mM}$ $\mathrm{Na}_{2} \mathrm{TeO}_{3}, 2 \mathrm{mM} \mathrm{CdCl}$, and $6 \mathrm{mM}$ MPA was prepared in a three-necked round-bottom flask, and the $\mathrm{pH}$ of the solution was adjusted to 11 using a small amount of $\mathrm{NaOH}$. A series of CdTe QDs were prepared at $90^{\circ} \mathrm{C}$ with different reaction times under microwave irradiation. A microwave chemical reactor (Green Motif I) from J. Science Lab Co., Ltd. (Kyoto, Japan), equipped with a controllable temperature unit, was used to synthesize CdTe QDs. The system works at a power of 30 to $300 \mathrm{~W}$ and operates at a frequency of $2450 \pm 30 \mathrm{MHz}$.

The synthesized QDs were precipitated with twice the volume of 2-propanol and the superfluous MPA and $\mathrm{Cd}^{2+}$ were removed by centrifugation at $10000 \mathrm{rpm}$ for $10 \mathrm{~min}$. The obtained powdered QDs were then dissolved in a Tris buffer solution for optical characterization. All optical measurements were performed at room temperature. UV-vis absorption spectra were obtained using a UV-2550PC spectrophotometer (Shimadzu, Japan). Photoluminescence (PL) spectra were recorded with a FP-7800 spectrofluorometer (JASCO, Japan). ECL spectra were recorded with a USB2000 fiber optic spectrofluorometer (Ocean Optics, USA).

\section{Results and Discussion}

Microwave synthesis of CdTe QDs with different particle sizes

Microwave heating can deliver energy to reacting species at a much greater rate than conventional heating, allowing one to more readily overcome activation barriers, and greatly reduces the reaction times. The following chemical reactions are proposed in the synthesis of CdTe QDs via the microwave irradiation reduction route:
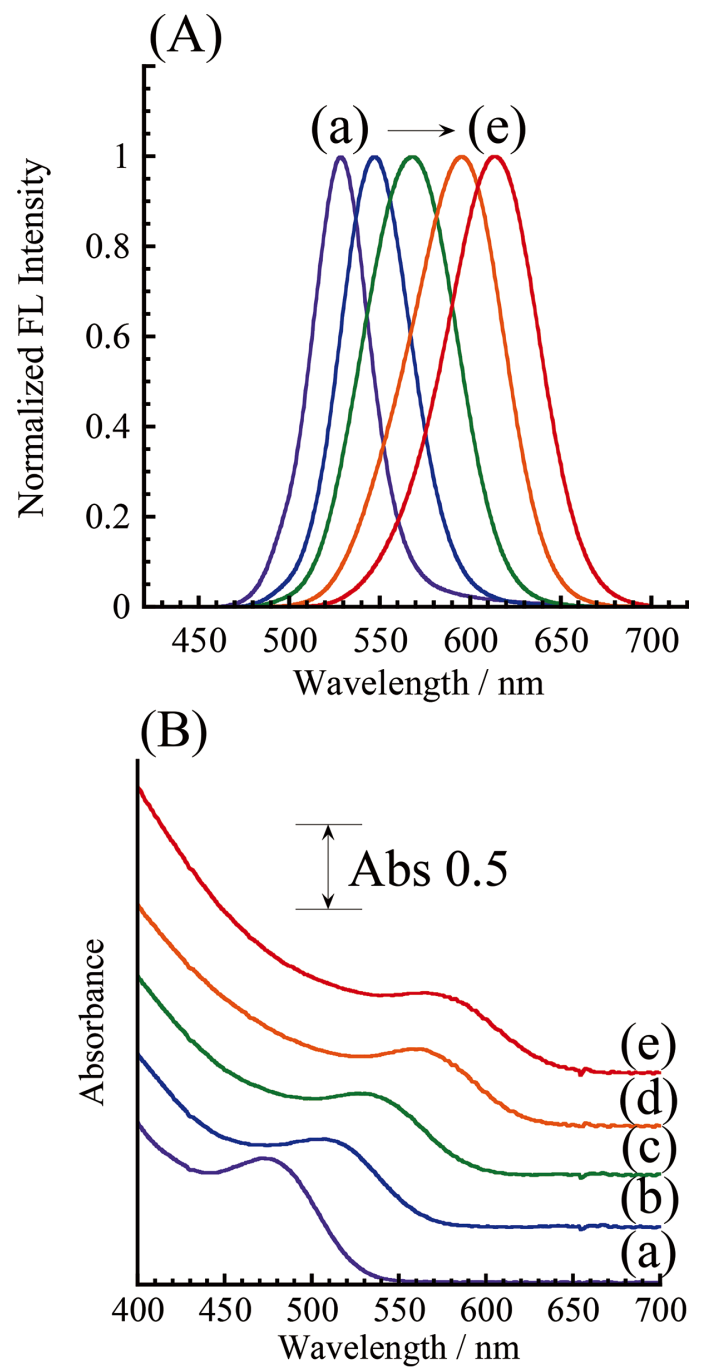

Fig. 1 Photoluminescence (PL) (A) and UV-visible absorption spectra (B) of MPA-capped CdTe QDs prepared with different reaction times of (a) 20, (b) 30, (c) 40, (d) 60 and (e) $150 \mathrm{~min}$. The diameters of the obtained CdTe QDs were (a) 1.3, (b) 2.3, (c) 2.8, (d) 3.2 and (e) $3.4 \mathrm{~nm}$, respectively. PL spectra were recorded with excitation at $380 \mathrm{~nm}$.

Scheme 1:

$$
\begin{aligned}
& \mathrm{TeO}_{3}{ }^{2-}+6 \mathrm{MPA} \longrightarrow \mathrm{Te}^{2-}+3 \mathrm{DTDPA}+3 \mathrm{H}_{2} \mathrm{O} \\
& \mathrm{CdCl}_{2}+y \mathrm{Te}^{2-}+x \mathrm{MPA} \longrightarrow \longrightarrow \\
& \mathrm{Cd}-(\mathrm{MPA})_{\mathrm{x}} \mathrm{Te}_{\mathrm{y}}(\text { precursor })+2 \mathrm{Cl}^{-}
\end{aligned}
$$

$$
\text { Cd-(MPA) } \text { Te }_{\mathrm{y}} \text { (precursor) } \longrightarrow \text { CdTe QDs }
$$

MPA severed as stabilizer as well as a weak reducing agent. Under microwave irradiation, $\mathrm{TeO}_{3}{ }^{2-}$ was reduced to $\mathrm{Te}^{2-}$ by MPA and MPA was oxidized to dithiodipropionic acid (DTDPA) accordingly, as is shown in Eq. (1). ${ }^{18}$ The precursors of CdTe QD were then formed by a reaction between freshly supplied $\mathrm{Te}^{2-}$ and $\mathrm{Cd}^{2+}$ in the presence of MPA (Eq. (2)). The precursors were converted to CdTe QDs by refluxing the reaction mixture at $90^{\circ} \mathrm{C}$ (Eq. (3)).

The formation of CdTe QDs was characterized by photoluminescence (PL) and UV-Vis absorption spectra (Fig. 1). The PL emission peak position of QDs shifts from $529 \mathrm{~nm}$ 

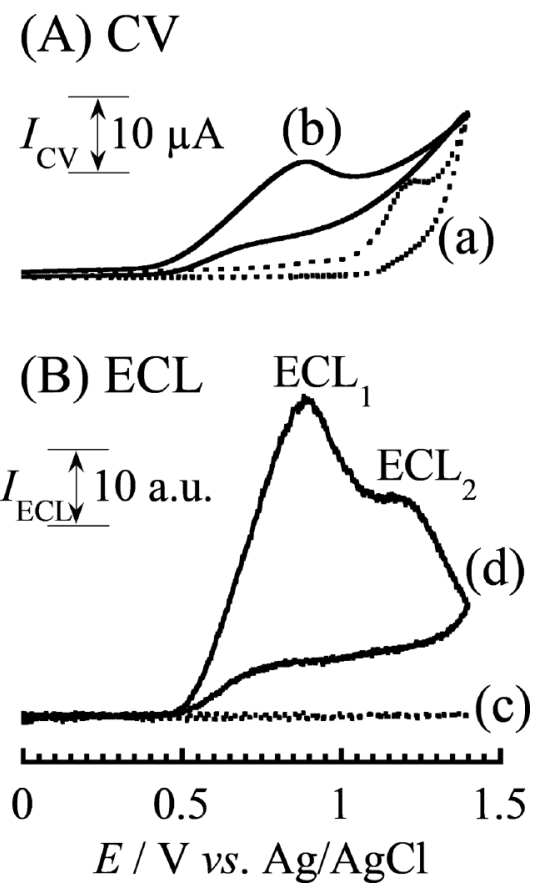

Fig. 2 Cyclic voltammograms (A) and the corresponding ECL responses (B) of $1.0 \mu \mathrm{M}$ CdTe QDs (3.2 nm in diameter) at GC electrode. The dotted curve (a) and solid curve (b) are CVs of $1 \mu \mathrm{M}$ CdTe QDs and $1 \mathrm{mM}$ TPrA in $0.1 \mathrm{M}$ Tris buffer solution ( $\mathrm{pH} 8.5$ ), respectively. The dotted curve (c) and solid curve (d) are the ECL responses for $1 \mu \mathrm{M}$ CdTe QDs observed in the absence and in the presence of $1 \mathrm{mM}$ TPrA in $0.1 \mathrm{M}$ Tris buffer solution $(\mathrm{pH} 8.5$ ), respectively. Potential scan rate was $50 \mathrm{mV} / \mathrm{s}$.

(green) to $614 \mathrm{~nm}$ (red) with an increase of the reaction time from 20 to $150 \mathrm{~min}$, and sharp PL peaks indicate a highly monodisperse sample. The particle sizes of the synthesized CdTe QDs were estimated to be 1.3, 2.3, 2.8, 3.2 and $3.4 \mathrm{~nm}$ according to the following empirical equation: ${ }^{19}$

$$
\begin{aligned}
& D(\mathrm{~nm})=\left(9.8127 \times 10^{-7}\right) \lambda^{3}- \\
&\left(1.7147 \times 10^{-3}\right) \lambda^{2}+1.0064 \lambda-194.84
\end{aligned}
$$

In Eq. (4), $D(\mathrm{~nm})$ is the diameter of $\mathrm{QD}$ and $\lambda(\mathrm{nm})$ is the wavelength of the first excitonic absorption peak of the UV-Vis absorption spectra. The molar extinction coefficient $(\varepsilon)$ of a CdTe QDs solution can be obtained from an empirical function of the nanocrystals size as follows: ${ }^{19}$

$$
\varepsilon\left(\mathrm{M}^{-1} \mathrm{~cm}^{-1}\right)=10043(D)^{2.12}
$$

\section{Anodic ECL of CdTe QDs/TPrA system}

Figure 2 shows cyclic voltammograms $(\mathrm{CV})$ and corresponding ECL responses of $1 \mu \mathrm{M}$ CdTe QDs (3.2 $\mathrm{nm}$ in diameter) at a GC electrode. In Fig. 2(A), the dotted curve (a) and solid curve (b) are CVs of $1 \mu \mathrm{M}$ CdTe QDs and $1 \mathrm{mM}$ TPrA in $0.1 \mathrm{M}$ Tris buffer solution ( $\mathrm{pH} 8.5)$, respectively. In Fig. 2(B), the dotted curve (c) is the ECL background for $1 \mu \mathrm{M}$ CdTe QDs. No ECL response could be observed without TPrA. With the addition of $1 \mathrm{mM}$ TPrA into the solution, however, two ECL signals appeared when the potential was scanned from 0 to $+1.4 \mathrm{~V} v s$. $\mathrm{Ag} / \mathrm{AgCl}$, as is shown in curve (d). The first ECL signal $\left(\mathrm{ECL}_{1}\right)$ was observed at around $+0.8 \mathrm{~V}$ vs. $\mathrm{Ag} / \mathrm{AgCl}$, where the direct oxidation of TPrA at the GC electrode occurs, and the second

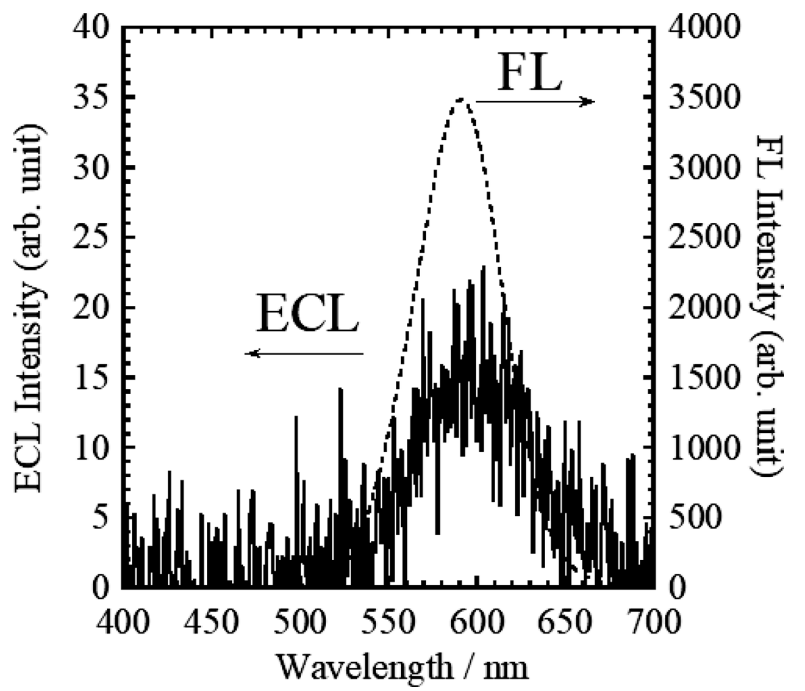

Fig. 3 ECL and PL spectra of CdTe QDs with diameter of $3.2 \mathrm{~nm}$. ECL spectrum was recorded by a continuous potential scan between 0 to $1.0 \mathrm{~V}$ vs. $\mathrm{Ag} / \mathrm{AgCl}$ in $0.1 \mathrm{M}$ Tris buffer solution $(\mathrm{pH} 8.5)$ containing $1 \mu \mathrm{M}$ CdTe QDs (in diameter) and $1 \mathrm{mM}$ TrPA. The potential scan rate was $50 \mathrm{mV} / \mathrm{s}$, and the integration time for the ECL spectrum was $1 \mathrm{~min}$. PL spectrum was measured with the condition as shown in Fig. 1.

ECL signal $\left(\mathrm{ECL}_{2}\right)$ was observed at $+1.25 \mathrm{~V}$ vs. $\mathrm{Ag} / \mathrm{AgCl}$ coincided with the oxidation potential of CdTe QDs. The intensities of both $\mathrm{ECL}_{1}$ and $\mathrm{ECL}_{2}$ were found to be linearly proportional to the concentration of TPrA up to $5 \mathrm{mM}$. Since there was no ECL signal to be detected in the absence of either TPrA or CdTe QDs, the ECL signals should be associated with a reaction between the species generated from TPrA and CdTe QDs. As is shown in Scheme 2, we believe that the $\mathrm{ECL}_{2}$ signal was emission of the excited CdTe QDs*, which was produced via the oxidative-reduction coreactant mechanism, as had been documented in the $\mathrm{Ru}(\mathrm{bpy})_{3}{ }^{2+} / \mathrm{TPrA}$ ECL system. ${ }^{14}$

Scheme 2:

$$
\begin{aligned}
& \mathrm{TPrA} \longrightarrow \mathrm{TPrA}^{\bullet+}+\mathrm{e}^{-} \rightleftharpoons \mathrm{TPrA}^{\bullet}+\mathrm{H}^{+} \\
& \mathrm{QD} \longrightarrow \mathrm{QD}^{\bullet+}+\mathrm{e}^{-} \\
& \mathrm{QD}^{\bullet+}+\mathrm{TPrA}^{\bullet} \longrightarrow \mathrm{QD}^{*}+\mathrm{TPrA} \text { fragments }
\end{aligned}
$$

In weak basic solutions, the electrochemical oxidation steps for TPrA involve a one-electron oxidation to form TPrA cation radicals $\left[\mathrm{TPrA}^{*+}=\left(\mathrm{CH}_{3} \mathrm{CH}_{2} \mathrm{CH}_{2}\right)_{3} \mathrm{~N}^{*+}\right]$, and the subsequent deprotonation at the $\alpha$-carbon to produce a strong reducing TPrA free radicals $\left[\mathrm{TPrA}{ }^{*+}=\left(\mathrm{CH}_{3} \mathrm{CH}_{2}\right)_{2} \mathrm{C}^{\cdot} \cdot \mathrm{HCH}_{2} \mathrm{CH}_{3}\right]$ in Eq. (6). ${ }^{14,16}$ On the other hand, CdTe QDs was oxidized at $+1.25 \mathrm{~V}$ to form an intermediate radical, CdTe $\mathrm{QD}^{+}$, at the electrode surface (Eq. (7)). ${ }^{12,13,20}$ The excited state species (CdTe QD*) was thus formed by the reaction of CdTe QD ${ }^{*+}$ with TPrA* in Eq. (8).

Clearly, the mechanism proposed above is not able to explain the reaction for $\mathrm{ECL}_{1}$ because $\mathrm{CdTe} \mathrm{QD}^{*+}$ could not be generated directly at this potential, other reactions that can be involved in the oxidation of TPrA should be taken into account. To confirm the emission species for $\mathrm{ECL}_{1}$, the ECL emission spectrum was measured, as is shown in Fig. 3. The spectrum was recorded during a potential scan from 0 to $1.0 \mathrm{~V} v s$. $\mathrm{Ag} / \mathrm{AgCl}$ in $0.1 \mathrm{M}$ 
Tris buffer solution ( $\mathrm{pH} 8.5$ ) containing $1 \mu \mathrm{M}$ CdTe QDs (3.2 nm in diameter) and $1 \mathrm{mM}$ TrPA with a scan rate of $20 \mathrm{mV} / \mathrm{s}$. Since the ECL emission closely matches the PL spectrum, it can be attributed to the same excited state as was observed in PL. We consider that the formation of strong reducing radicals $\operatorname{TPrA}^{\bullet}\left(E^{\circ} \approx-1.7 \mathrm{~V}\right.$ vs. $\left.\mathrm{Ag} / \mathrm{AgCl}^{15}\right)$ in Eq. (6) is sufficient to reduce CdTe QD to a CdTe QD*- radical anion $\left(E^{\circ} \approx-1.32 \mathrm{~V}\right.$ vs. $\mathrm{Ag} / \mathrm{AgCl}$, which is determined by cyclic voltammetry, shown in Fig. S1). The $\operatorname{TPrA} A^{++}$radical cation formed in Eq. (6) is a sufficient stable intermediate with a halflife of $\sim 0.5 \mathrm{~ms},{ }^{21}$ which can serve as an oxidant to oxidize CdTe $\mathrm{QDs}^{-}$in this system. The possible route for $\mathrm{ECL}_{1}$ is summarized in Scheme 3, which involves the formation of CdTe QDs excited state by the reaction of TPrA ${ }^{*+}$ with CdTe QDs*.

Scheme 3:

$$
\begin{aligned}
& \mathrm{TPrA} \longrightarrow \mathrm{TPrA}^{\bullet+}+\mathrm{e}^{-} \rightleftharpoons \mathrm{TPrA}^{\bullet}+\mathrm{H}^{+} \\
& \mathrm{QD}^{+} \mathrm{TPrA} \bullet \longrightarrow \mathrm{QD}^{\bullet-}+\mathrm{TPrA} \text { fragments } \\
& \mathrm{QD}^{\bullet-}+\mathrm{TPrA}^{\cdot+} \longrightarrow \mathrm{QD}^{*}+\mathrm{TPrA} \text { fragments }
\end{aligned}
$$

The ECL intensity of the CdTe/TPrA system strongly depends on the solution $\mathrm{pH}$. ECL intensity of $\mathrm{ECL}_{2}$ was found to be increased significantly with increasing of $\mathrm{pH}$ values, whereas the intensity of $\mathrm{ECL}_{1}$ was decreased when the $\mathrm{pH}$ was higher than 9. Tertiary amine radical cations are generally believed to be short-lived intermediates, and the dissociation constant, $\mathrm{p} K_{\mathrm{a}}$, of TPrA ${ }^{\bullet+}$ is assumed to be $\sim 8.0 .^{22}$ Increasing the $\mathrm{pH}$ would lead to decrease the fraction of $\operatorname{TPr}{ }^{\cdot+}$ at the electrode surface, and consequently decrease the intensity of $\mathrm{ECL}_{1}$. The experiment results support the reaction routes proposed in Scheme 3.

\section{Size dependent effect and the correlation between ECL properties} and electronic energy states of CdTe QDs

As displayed in Fig. 1, the wavelengths of the fluorescence and absorption can be tuned by the size CdTe QDs due to the variation of the energy band gap $E_{\mathrm{g}}$; it was of our interest to elucidate how the size of CdTe QDs or $E_{\mathrm{g}}$ correlate with their ECL properties. Figure 4 shows the dependence of the intensities of $\mathrm{ECL}_{1}$ and $\mathrm{ECL}_{2}$ on the particle size. The concentrations of CdTe QDs were fixed as $1 \mu \mathrm{M}$, while the particle sizes of CdTe QDs being examined were 1.3, 2.3, 2.8, 3.2 and $3.4 \mathrm{~nm}$. The values of $E_{\mathrm{g}}$ of these particles were estimated to 2.35, 2.18, 2.09, 2.02 and $1.93 \mathrm{eV}$ from the onset of absorption of UV-Vis spectra according to Fendler et al.'s method. ${ }^{23}$ Intensities of both $\mathrm{ECL}_{1}$ and $\mathrm{ECL}_{2}$ increased with increasing of particle size. It is worth noting that the diameter of CdTe QDs has a considerable influence on the response of $\mathrm{ECL}_{1}$. The signal of $\mathrm{ECL}_{1}$ was not detectable when the diameter of CdTe QDs was less than $2.4 \mathrm{~nm}$, and the relative intensity of $\mathrm{ECL}_{1}$ (intensity ratio of $\mathrm{ECL}_{1}$ and $\mathrm{ECL}_{2}$ ) increased significantly with increasing of the particle size.

In considering the reaction for $\mathrm{ECL}_{1}$, the sufficient energy is required in the electron-transfer reaction of in Eq. (10) to produce the lowest excited state species CdTe QDs*. The energy available between $\operatorname{TPrA}^{*+}$ with $\mathrm{QD}^{--}$can be calculated from the enthalpy change ( $\Delta H$ in $\mathrm{eV}$ ) by means of the following equation:

$$
-\Delta H=\left(E_{\text {TPA }+/ \text { TPA }}^{\circ \prime}-E_{\text {CdTe/CdTe-- }}^{\circ}\right)-0.1(\mathrm{eV})
$$

where $E_{\text {TPA +/TPA }}^{\circ \prime}$ and $E_{\text {CdTe/CdTe-- }}^{\circ \prime}$ are the oxidation formal potential of the TPrA and the reduction formal potential of QDs,

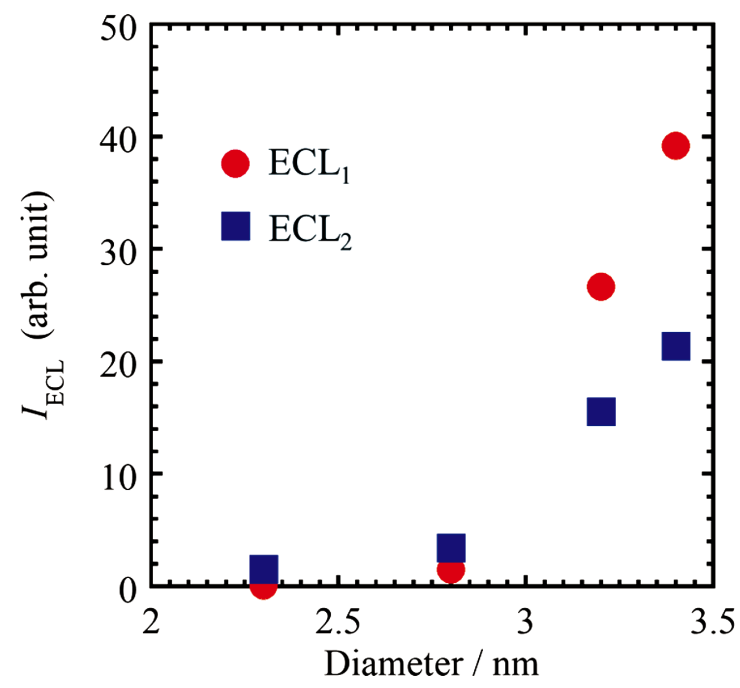

Fig. 4 Dependence of signals for $\mathrm{ECL}_{1}$ and $\mathrm{ECL}_{2}$ on the particle size in CdTe QDs/TPrA ECL system. The diameters of CdTe QDs were $1.3,2.3,2.8,3.2$ and $3.4 \mathrm{~nm}$. The other conditions were the same as in Fig. 2.

$0.1 \mathrm{eV}$ is an estimate of the temperature entropy approximation term $(\mathrm{T} \Delta S)$ at $25^{\circ} \mathrm{C} . .^{24}$

QDs have discrete energy levels and are expected to undergo electron transfer, mediated through the valence band (VB) edge and the conduction band (CB) edge. $E_{\mathrm{g}}$ basically corresponds to the transitions gap between the VB and the $\mathrm{CB}$ energy states, while PL or ECL emission originate from the excitonic transition due to radiative recombination of the electron in an excited energy level $E_{\mathrm{s}}$ with the hole in the VB. From the results of PL and ECL measurements, we believe that the electron in a lowest excited state $E_{\mathrm{s}}$ located a little above the bottom of the $\mathrm{CB}$ in CdTe QDs. The apparent band gap of QDs measured by PL emission may deviate from the VB-CB transition energy $E_{\mathrm{g}}$, due to the impurity levels or the surface effects of QDs. In a case of QDs with a diameter of $3.4 \mathrm{~nm}$, the lowest excited state energy $\left(E_{\mathrm{s}}\right)$ of was determined to be as $2.02 \mathrm{eV}$ from the max emission wavelength at PL spectrum. $E^{\circ \prime}{ }_{\text {TPA.+/TPA }}$ and $E_{\text {CdTe/CdTe-- were }}^{\circ{ }^{\prime}}$ determined to be $+0.85 \mathrm{~V}$ vs. $\mathrm{Ag} / \mathrm{AgCl}$ and $-1.35 \mathrm{~V}$ by cyclic voltammetry. $-\Delta H$ in Eq. (10) was calculated to be $2.10 \mathrm{eV}$, which is larger than $E_{\mathrm{s}}(2.02 \mathrm{eV})$ of CdTe QDs. Hence, CdTe QDs* could be directly generated. ${ }^{23}$ A cathodic-shift of $E^{\circ \prime}$ CdTe/CdTe- is observed in the CV experiment with a decrease of the diameter of QDs. In a case of QDs with diameter of $2.4 \mathrm{~nm}$,

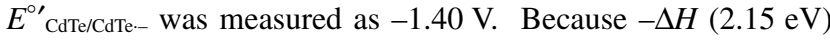
was less than $E_{\mathrm{s}}(2.26 \mathrm{eV})$ of CdTe QDs, signal of $\mathrm{ECL}_{1}$ was not detected in the present system. The results in this study show that ECL intensity of CdTe QDs increased with the particle sizes. The increment in the ECL intensity with increasing in particle size was possibly attributed to the energy involved in the reactions of Eqs. (8) and (10). The smaller is $E_{\mathrm{g}}$ with a larger particle size, the more is the ECL intensity. The other effects such as stability and surface properties also show influences on ECL properties, which are under investigation in the further study.

\section{Conclusions}

CdTe QDs with different sizes were successfully synthesized with a microwave-assisted hydrothermal method, and were 
studied as ECL probes using TPrA as a coreactant. We demonstrated that CdTe QDs could be excited in the anodic potential range to give two ECL signals at potentials corresponding to the oxidation of TPrA and direct oxidation of CdTe QDs, respectively, which exhibited the size-dependent characteristics. We found that ECL of the CdTe QDs/TPrA system occurred more easily using a bigger particle size with a relatively smaller band gap energy, because the chemical energy of the electron transfer reaction between the intermediate radicals should match the band gap energy of QDs. This may intrigue researchers into gaining a new interest when investigating the ECL property of QDs and promote the step of their applications for analytical purpose.

\section{Acknowledgements}

We appreciate the kind support from Japan Society for the Promotion of Sciences (JSPS), Grant-in-Aid for Scientific Research (C) (No. JP16K05813) and Prof. J. S. Y. acknowledge the support from the Science and Technology Program of Guangdong Province (No. 2018A050506006).

\section{Supporting Information}

This material is available free of charge on the Web at http:// www.jsac.or.jp/analsci/.

\section{References}

1. C. B. Murray, D. J. Norris, and M. G. Bawendi, J. Am. Chem. Soc., 1993, 115, 8706.

2. R. Gill, R. Freeman, J. P. Xu, I. Willner, S. Winograd, I. Shweky, and U. Banin, J. Am. Chem. Soc., 2006, 128, 15376.

3. Z. Zhelev, R. Bakalova, H. Ohba, R.Jose, Y. Imai, and Y. Baba, Anal. Chem., 2006, 78, 321.
4. T. Jin, F. Fujii, H.Sakata, M. Tamura, and M. Kinjo, Chem. Commun., 2005, 34, 4300.

5. K.-Y. Yi and C.-S. Wei, Int. J. Electrochem. Sci., 2017, 12, 3472 .

6. Y. Shi, L. Liu, H. Pang, H. Zhou, G. Zhang, Y. Ou, X. Zhang, J. Du, and W. Xiao, Nanoscale Res. Lett., 2014, 9, 115 .

7. H. Liang, D. Song, and J. Gong, Biosens. Bioelectron., 2014, 53, 363 .

8. H. Y. Han, Z. H. Sheng, and J. G. Liang, Anal. Chim. Acta, 2007, 596, 73 .

9. S. N. Ding, J. J. Xu, and H. Y. Chen, Chem. Commun., 2006, 34, 3631 .

10. R. Russell, A. J. Stewart, and L. Dennany, Anal. Bioanal. Chem., 2016, 408, 7129.

11. G. F. Jie, P. Liu, L. Wang, and S. S. Zhang, Electrochem. Commun., 2010, 12, 22

12. H. J. Liu, J. Lei, and H. Ju, Anal. Chem., 2007, 79, 8055.

13. L. H. Zhang, X. Q. Zou, E. Ying, and S. J. Dong, J. Phys. Chem. C, 2008, 112, 4451 .

14. Y. $\mathrm{Zu}$ and A. J. Bard, Anal. Chem., 2000, 72, 3223.

15. R. Y. Lai and A. J. Bard, J. Phys. Chem. A, 2003, 107 , 3335.

16. F. Takahashi and J. Jin, Electroanalysis, 2008, 20, 1581.

17. H. Wang, Q. Chen, Z. Tan, X. Yin, and L. Wang, Electrochim. Acta, 2012, 72, 28.

18. C. C. Reinhart and E. Johansson, Chem. Mater., 2015, 27, 7313.

19. W. Yu, L. Qu, W. Guo, and X. Peng, Chem. Mater, 2003 , $15,2854$.

20. H. Jiang and X.-M. Wang, Electrochem. Commun., 2009, 127, 1207.

21. W. Miao, Chem. Rev., 2008, 108, 2506.

22. S. Das and C. V. Sonntag, Z. Naturforsch. B, 1986, 41, 505.

23. Y. Tian, T. Newton, N. A. Kotov, D. M. Guldi, and J. H. Fendler, J. Phys. Chem., 1996, 100, 8927.

24. A. J. Bard, "Electrogenerated Chemiluminescence", 2004 Marcel Dekker, Inc., New York, 12. 